\title{
Prevalence of hypertension in the rural adult population of Osun State, southwestern Nigeria
}

This article was published in the following Dove Press journal:

International Journal of General Medicine

24 April 2013

Number of times this article has been viewed

\section{EO Asekun-Olarinmoye' \\ PO Akinwusi ${ }^{2}$ \\ WO Adebimpe' \\ MA Isawumi ${ }^{3}$ \\ MB Hassan ${ }^{3}$ \\ OA Olowe ${ }^{4}$ \\ OB Makanjuola ${ }^{4}$ \\ CO Alebiosu ${ }^{2}$ \\ TA Adewole ${ }^{5}$ \\ 'Department of Community \\ Medicine, ${ }^{2}$ Department of Medicine, \\ ${ }^{3}$ Department of Surgery, ${ }^{4}$ Department \\ of Microbiology, ${ }^{5}$ Department of \\ Chemical Pathology, College of Health \\ Sciences, Osun State University, \\ Osogbo, Osun State, Nigeria}

Correspondence: EO Asekun-Olarinmoye Department of Community Medicine, College of Health Sciences,

Faculty of Clinical Sciences,

Osun State University, PMB 4494,

Oke-Baale, Osogbo, Osun State, Nigeria

$\mathrm{Tel}+2348033602$ I84

Email esther.asekun-olarinmoye@uniosun. edu.ng
Background: The purpose of this study was to determine the prevalence of hypertension in two rural communities of Osun State, Nigeria.

Methods: A consenting adult population of the Alajue and Obokun rural communities in southwestern Nigeria that presented for the screening exercise participated in this communitybased cross-sectional descriptive study. Two hundred and fifty-nine respondents aged older than 18 years completed a standardized, pretested, structured questionnaire as part of activities celebrating World Kidney Day and World Glaucoma Day in 2011. Anthropometric data and blood pressure were recorded, and the data were analyzed using the Statistical Package for Social Sciences version 17.

Results: The mean age of the respondents was $49.7 \pm 1.6$ years, 100 (38.6\%) were males, $84(32.4 \%)$ were farmers, and $111(42.9 \%)$ were traders. The prevalence of hypertension was $13.16 \%$ (present in 34 respondents). Seventeen (6.6\%) had isolated systolic hypertension, while $11(4.2 \%)$ had isolated diastolic hypertension. Two hundred and thirty-six (91.1\%) undertook daily exercise lasting at least 30 minutes and 48 (18.5\%) had ever taken antihypertensive drugs on a regular basis. Four respondents $(1.6 \%)$ claimed a family history of hypertension. The average body mass index (BMI) among respondents was $23.4 \pm 4.9 \mathrm{~kg} / \mathrm{m}^{2}, 51(19.6 \%)$ had a BMI of 25.0-29.9, and 30 (11.5\%) had a BMI $\geq 30$. A significant association existed between age older than 40 years and having hypertension $(P<0.05)$, while no relationship existed between age and BMI or between gender and hypertension $(P>0.05)$. Rates of older age and high BMI were significantly higher among hypertensives than among normotensives. Respondents with $\mathrm{BMI} \geq 25$ had at least a three times greater likelihood of developing hypertension than those with BMI $<25$ (odds ratio 2.9, 95\% confidence interval 0.007-0.056, $P=0.011$ ).

Conclusion: The prevalence of hypertension is high in this study population and we recommend scaling up primary prevention efforts to reduce this in Nigerian communities.

Keywords: prevalence, hypertension, anthropometry, body mass index, rural community

\section{Introduction}

Like many other noncommunicable diseases, hypertension is gradually assuming epidemic dimensions with the dawn of epidemiological transition. It is the most common cardiovascular disorder, affecting approximately one billion people globally and causing approximately 7.1 million deaths annually. ${ }^{1}$ Hypertension is the most common cardiovascular disease in Africans, ${ }^{2}$ and congestive cardiac failure is its commonest complication. ${ }^{3}$ Other complications include stroke, renal failure, atherosclerotic cardiovascular disease, and death. ${ }^{4}$ According to the World Health Organization, cardiovascular diseases are the leading cause of mortality and morbidity in developed countries and are emerging as a prominent public health problem in 
developing countries. ${ }^{5}$ In Nigeria, common cardiovascular diseases reported with a high incidence include heart failure, hypertension, and congenital heart disease, with hypertension being the commonest. ${ }^{6}$

The Nigerian National Noncommunicable Disease Survey in 1997 reported an adult hypertension prevalence of $11.4 \%$, with a variation of $14.8 \%$ and $9.8 \%$ in urban and rural areas, respectively. ${ }^{7}$ The cutoff value of a blood pressure (BP) 160/95 $\mathrm{mmHg}$ for hypertension was used, so giving an underestimation of the prevalence of hypertension. This would have been higher if the new cutoff value of $140 / 90 \mathrm{mmHg}$ was used. The prevalence of hypertension in both rural and urban communities in Nigeria is increasing. Various community surveys in Nigeria have shown an adult prevalence of $15 \%-36.6 \% .^{8-11}$ Oladapo et al recently reported a prevalence of $20.8 \%$ in the rural community of Egbeda, southwest Nigeria. ${ }^{12}$ The prevalence in semiurban Ile-Ife was $36.6 \%$, while that in Ipetumodu, Osun State, Nigeria, was $26.4 \%$ for mild hypertension ( $\mathrm{BP} \geq 140 / 90 \mathrm{mmHg}$ ) and $11.8 \%$ for moderate hypertension ( $\mathrm{BP} \geq 160 / 100 \mathrm{mmHg}$ ). ${ }^{12}$

The overall prevalence of hypertension ranges from $14.5 \%$ in Nigeria, $16.9 \%$ in Cameroon, $28.7 \%$ in Accra, ${ }^{13}$ to $32.6 \%$ for blacks in the United States. ${ }^{14}$ Cross-migration from rural to urban areas and vice versa, the asymptomatic nature of the disease, changing lifestyles, and the environment are contributing to the rising problem of hypertension. Hypertension has been associated with various factors, including age, gender, family history, alcohol consumption, smoking, obesity, level of education, and occupation. ${ }^{15}$

Hypertension is a silent killer and most patients are detected to have it incidentally when they are admitted to hospital for unrelated disease or subjected to pre-employment or preoperative medical checkups. Many patients are also diagnosed when they seek medical advice because of target organ damage. Target organ damage and associated clinical conditions are very common in newly diagnosed hypertensive patients in Nigeria, with a recently reported prevalence of $66.7 \% .{ }^{16}$ However, the majority of the rural population in Nigeria is marginalized and has inadequate access to health care and quality education. ${ }^{17} \mathrm{~A}$ significant number of rural dwellers seek consultations with indigenous and private health care practitioners, where regular screening for hypertension is not practiced. Clinic-based (opportunistic) screening of hypertension may not detect a large proportion of adults with hypertension. Community-based screening can improve the detection and treatment of hypertension. Few studies have demonstrated the occurrence of hypertension and its predisposing risk factors in rural areas of Nigeria.
The objective of this study was to determine the prevalence of hypertension in two selected rural communities in southwestern Nigeria.

\section{Materials and methods}

This was a community-based, descriptive, cross-sectional study carried out in two rural communities in Osun state in southwestern Nigeria. The two rural communities were Alajue and Ibokun, with a total population of 5000 people. The majority of the dwellers there are farmers and many are traders. Factors predisposing to a western lifestyle appeared minimal in these communities, although they have roads making the communities accessible to town and cities. All members of the communities aged 18 years and older constituted the target population.

This study was carried out as a part of a community-based screening program to raise awareness of the 2011 World Kidney Day celebrations. Entry commenced with holding a series of meetings with kings, traditional chiefs, politicians, heads of households, and other gatekeepers in the community. The rationale for the screening exercise was communicated to community members at these meetings. Community town hall meetings were organized for each of the communities to mobilize and sensitize community members further. At these meetings, community members eligible for screening were encouraged to come out en masse, with the assurance that all those who came out and consented to the exercise would be screened. The two communities were mobilized and sensitized regarding the importance of screening for noncommunicable diseases, in particular, hypertension and diabetes. All consenting adults who participated in the screening exercise had their BP recorded, in addition to anthropometric measurements. An interviewer administered a pretested questionnaire to collect basic data pertaining to sociodemographic characteristics and risk factors for hypertension among the respondents.

Ethical approval to conduct the study was obtained from the ethical research committee of Osun State University College of Health Sciences. A limitation of this study was its inability to study complications associated with hypertension in these rural communities, although this was actually beyond the scope of the present study.

\section{Training of survey team}

To standardize survey measurements and procedures, ten research assistants were trained using specially prepared survey manuals that conformed to recommended noncommunicable disease survey protocols. ${ }^{18}$ Before 
the main study, a field test was carried out to fine-tune instruments and procedures.

\section{Operational definitions}

Hypertension was defined as systolic BP $\geq 140 \mathrm{mmHg}$ and/or diastolic BP $\geq 90 \mathrm{mmHg}$, based on the guidelines of the International Society of Hypertension/World Health Organization $^{19}$ and the Seventh Joint National Committee on Hypertension, ${ }^{20}$ or being on regular antihypertensive drugs. Overweight/obesity was defined as BMI $\geq 25 \mathrm{~kg} / \mathrm{m}^{2}$ and $\geq 30 \mathrm{~kg} / \mathrm{m}^{2}$, respectively. A standardized calibrated mercury column type sphygmomanometer, stethoscope, common weighing machine, and measuring tape were used for physical examination.

\section{Measurements}

Informed verbal consent was obtained before measuring BP. The subjects rested quietly for 10 minutes, and BP was measured in the sitting posture with an appropriate-sized cuff encircling the arm. With the left arm of the subject held at the level of the heart, the maximum inflation level was determined using a mercury sphygmomanometer, using a $15 \mathrm{~cm}$ stethoscope and a cuff of appropriate size (a cuff of larger width was used for large arms).

BP was measured to the nearest $2 \mathrm{mmHg}$ on two occasions at an interval of one minute. BP measurements were made using the left arm of each study subject. Subjects were not permitted to smoke. In cases where the two readings differed by over $10 \mathrm{mmHg}$, a third reading was obtained, and the three measurements were averaged. The pressures at which sounds appeared and disappeared were taken as systolic BP and diastolic BP, respectively. BP was classified as normal, hypertensive, or isolated systolic or diastolic hypertension.

Body weight was measured (to the nearest $0.5 \mathrm{~kg}$ ) with the subject standing motionless on the calibrated weighing scale, with feet about $15 \mathrm{~cm}$ apart and weight equally distributed on each leg. Subjects were instructed to wear minimum outerwear (as culturally appropriate) and no footwear while the weight was being measured. Height was measured (to the nearest $0.5 \mathrm{~cm}$ ) using a calibrated tape and with the subject standing in an erect position against a vertical surface, and the head positioned so that the top of the external auditory meatus was level with the inferior margin of the bony orbit.

\section{Calculations}

BMI was calculated as weight in kilograms divided by height in meters squared. Based on their BMI, individuals were classified into four groups, ie, thin (BMI < 18.5), normal
(BMI 18.5-24.9), overweight (BMI 25.0-29.9), or obese $(\mathrm{BMI}>30.0)$, according to WHO classification.

\section{Data management}

The Statistical Package for Social Sciences (SPSS) software version 17 (SPSS Inc, Chicago, IL, USA) was used for data entry and analysis. Validity of data was ensured by double entry and random checks for errors and outlier values. Relevant frequency distribution tables and summary indices were generated. The Chi-square test was used to demonstrate relationships between categorical variables. Multivariate logistic regression analysis was done using systolic and diastolic $\mathrm{BP}$ as the dependent variable and risk factors identified to be significant in the bivariate analysis as independent variables. The level of significance was set at $P$ values $\leq 0.05$ for all inferential analysis of categorical variables.

\section{Results}

Table 1 shows that the age group 50-69 years constitutes the highest proportion $(\mathrm{n}=109,42.0 \%)$ among respondents. The mean age of the respondents was $49.7 \pm 1.6$ years. One hundred (38.6\%) were males, $111(42.9 \%)$ were traders, and $84(32.4 \%)$ were farmers by occupation. Table 2 shows that the prevalence of hypertension was $13.16 \%$ (34 respondents). Seventeen (6.6\%) had isolated systolic hypertension, 11 (4.2\%) had isolated diastolic hypertension, and 189 (73.0\%) were normotensive. Two hundred and thirty-six (91.1\%) undertook daily exercises lasting at least 30 minutes. Forty-eight (18.5\%) had ever taken antihypertensive drugs, mostly the centrally acting agent, alpha methyldopa, and the diuretic combination of amiloride and hydrochlorothiazide. Four (1.6\%) claimed a family history of hypertension and $48(18.5 \%)$ had ever taken antihypertensive drugs on a regular basis.

Table I Sociodemographic data of respondents

\begin{tabular}{lll}
\hline Variables $(\mathbf{n}=\mathbf{2 5 9})$ & Frequency & Percentage \\
\hline Age group, years & & \\
10-29 & 36 & 13.9 \\
$30-49$ & 75 & 29.0 \\
$50-69$ & 109 & 42.0 \\
$70-89$ & 38 & 14.6 \\
$\geq 90$ & 1 & 0.4 \\
Gender & & \\
Male & 100 & 38.6 \\
Females & 159 & 61.4 \\
Occupation & & \\
Trading & 111 & 42.9 \\
Farming & 84 & 32.4 \\
Artisan & 18 & 6.9 \\
Others & 46 & 17.8 \\
\hline
\end{tabular}


Table 2 Pattern of hypertension in rural communities

\begin{tabular}{lll}
\hline Blood pressure $\mathbf{( m m H g})$ & Frequency & Percentage \\
\hline Normal & 189 & 73.0 \\
Hypertension & 34 & 13.1 \\
Systolic hypertension & 17 & 6.6 \\
Diastolic hypertension & 11 & 4.2 \\
Nonrecordable & 8 & 3.1 \\
\hline
\end{tabular}

The prevalence of overweight and obesity in the study population was as follows: 51 respondents $(19.6 \%)$ had a BMI of 25.0-29.9 while $30(11.5 \%)$ had a BMI of $\geq 30$. Mean BMI in the respondents was $23.4 \pm 4$.9. Figure 1 shows that more females $(n=19)$ were hypertensive as compared with males $(n=15)$. Similarly, more females were overweight or obese ( $n=35$ and $n=19$, respectively) than males ( $n=16$ and $\mathrm{n}=11)$. A significant association existed between age older than 40 years and having hypertension $(P<0.05)$, while no relationship existed between age and BMI or between gender and hypertension $(P>0.05)$. Respondents with $\mathrm{BMI} \geq 25$ had a three times greater likelihood of developing hypertension compared with those having a BMI $<25$ (odds ratio $2.9,95 \%$ confidence interval 0.007-0.056, $P=0.011$ ). Older age and increased BMI were significantly more common in hypertensive respondents than in their normotensive counterparts.

\section{Discussion}

The mean age of the respondents was about 49 years. This compares well with another study showing a mean age of about 42 years in rural communities in Nigeria. ${ }^{21}$ Just over $10 \%$ of the rural respondents we screened were hypertensive. This is consistent with the finding of a $10 \%$ prevalence in a typical

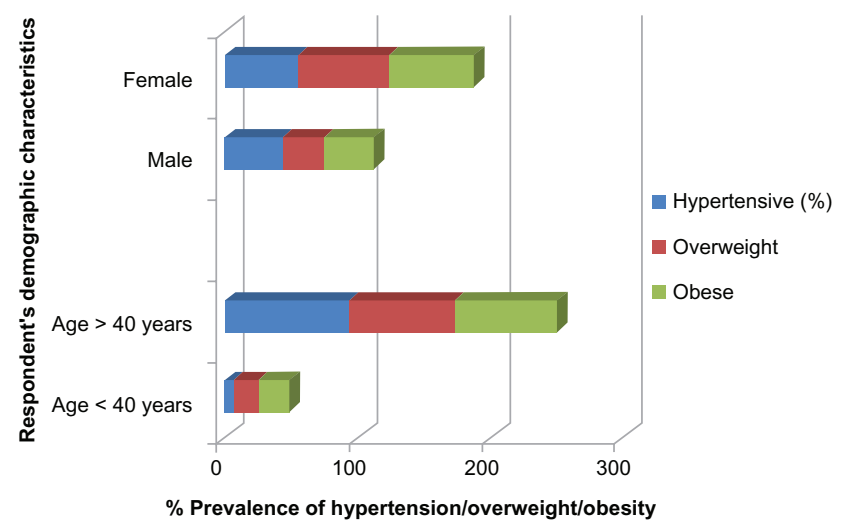

Figure I Association between demographic characteristics and hypertension and BMI.

Notes: Statistics: Age vs hypertension: $x^{2}=16.274$, df 3, P 0.00I; Age vs BMI: $x^{2}$ 1.608, df 2, $P$ 0.448. Sex vs hypertension: $x^{2}=2.904$, df $3, P$ 0.04; Sex vs BMI: $x^{2}$ 1.608 , df 2, $P 0.448$.

Abbreviation: BMI, body mass index. community in rural India. ${ }^{22}$ However, the prevalence in our study is lower than that reported by other studies from Nigeria (14.5\%) and Cameroon $\left(16.9 \%,{ }^{23} 18.3 \%,{ }^{24}\right.$ and $\left.19.04 \%\right) .{ }^{25}$ It is also much lower when compared with other studies reporting prevalences of $25.4 \%,{ }^{21} 28.3 \%,{ }^{13} 28.7 \%,{ }^{26} 30.5 \%,{ }^{27}$ and $37.7 \%{ }^{28}$ However, our figure is slightly higher than that from a study conducted in Sudan, which reported a prevalence of 7.5\% ${ }^{29}$ In this study, about $6.6 \%$ had isolated systolic and $4.2 \%$ diastolic hypertension, which is similar to another study reporting isolated systolic hypertension in $10.5 \%$ and isolated diastolic hypertension in $9.0 \%$ of subjects. ${ }^{30}$

A diverse pattern in the prevalence of hypertension can be said to exist in rural populations around the world. Typical rural populations, being marginalized and vulnerable communities in Nigeria, show a pattern of people migrating to urban areas or still living rurally but working in urban areas. In the process, some may be adopting the western lifestyle in the form of diet and physical activity related to urbanization and modernization, and western lifestyle has been identified as a risk factor for hypertension..$^{31}$ Like many other developing countries, disparity exists between rural and urban populations in terms of health facilities, education, and economic pursuits. ${ }^{15}$ For the same reason, about $10 \%$ of our rural population had taken antihypertensive drugs, mostly methyldopa and a combination of amiloride and hydrochlorothiazide, and were not on newer antihypertensive drugs for reasons of affordability and accessibility. Further, the primary health care centers that serve this rural population only stock first-line antihypertensive drugs requiring minimal biochemical monitoring. This changing epidemiology may be affecting the pattern of hypertension found in Africa.

A BP survey of 20 rural Ghanaian villages in 1973 found a hypertension prevalence of $2 \%-5 \%$, and concluded that hypertension was not a significant health problem in rural Ghanaians. ${ }^{32}$ Thus, an increase in the prevalence of hypertension can be said to be occurring in rural populations undergoing modernization. This supports the fact that hypertension, which was considered to be nonexistent or extremely rare in most African societies, particularly in rural communities, is now emerging as a public health problem in sub-Saharan Africa, ${ }^{32,33}$ and its prevalence will increase even further unless broad and effective preventive measures are implemented. ${ }^{34}$ There is a need for primary prevention efforts targeted to all communities, both rural and urban. Furthermore, the clinical significance of our findings is that, given that hypertension is now on the rise in rural areas of southwestern Nigeria, and probably related to the recent rise in occurrence of modifiable risk factors of hypertension 
occasioned by adoption of lifestyle changes in these rural populations, it is imperative that health policy-makers give priority to improved allocation of health resources to rural health facilities for effective management of hypertension.

In this study, hypertension was associated with advancing age. This supports a study in which hypertension has been found to be associated with older age,${ }^{29}$ and yet others finding an association in people older than 40 years..$^{25,35-38}$ It had been reported that the age-standardized prevalence of hypertension is significantly higher in urban than in rural areas for both men and women. ${ }^{39}$ In this study, hypertension was associated with being overweight and obese, and corroborates similar studies showing that BMI is strongly and independently associated with systolic and diastolic BP. ${ }^{40-42}$ In fact, overweight and obesity are no longer rare in rural residents. ${ }^{13,43}$

\section{Conclusion}

The prevalence of hypertension is on the increase in rural areas, contrary to speculation, and is associated with certain sociodemographic factors and BMI. Hence, there is a need for primary prevention efforts targeted at modifiable risk factors on a large scale.

\section{Acknowledgments}

The authors wish to thank the kings and members of the communities where the data were collected. We also thank our research secretariat for their efforts, and the ethical review committee of Osun State University for granting approval for us to conduct this study.

\section{Disclosure}

The authors report no conflicts of interest in this work.

\section{References}

1. Brundtland GH. From the World Health Organization. Reducing risks to health, promoting healthy life. JAMA. 2002;288:1974.

2. Akinkugbe OO. World epidemiology of hypertension in blacks. In: Hall WD, Saunders E, Shulman NB, editors. Hypertension in Blacks. Philadelphia, PA: Chicago Year Book Publishers; 1985.

3. Akinkugbe OO. High Blood Pressure in the African. Edinburg, Scotland: Churchill Livingstone; 1972.

4. Bloch MJ, Basile JN. Hypertension in the elderly. In: Black HR, Elliot WJ, editors. Hypertension: A Companion to Braunwald's Heart Disease, 1st ed. Philadelphia, PA: Saunders: 2007.

5. World Health Organization. The World Health Report-Reducing Risks, Promoting Healthy Lifestyles. Geneva, Switzerland: World Health Organization; 2002.

6. Mukadas AO, Misbau U. Incidence and patterns of cardiovascular disease in north western Nigeria. Niger Med J. 2009;50:55-57.

7. Akinkugbe OO, editor. National Expert Committee on Non-Communicable Diseases in Nigeria. Final Report of National Survey. Lagos, Nigeria: Federal Ministry of Health and Social Services; 1997.

8. Akinkugbe OO. Current epidemiology of hypertension in Nigeria. Arch Ibad Med. 1999;1:3-4.
9. Akinkugbe OO. Non-communicable disease, the next epidemic: Nigeria's preparedness. Nig J Clin Pract. 2000;3:37-42.

10. Kadiri S. Tracking cardiovascular disease in Africa. BMJ. 2005;331: 711-712.

11. Adedoyin RA, Mbada CE, Balogun MO, et al. Prevalence and pattern of hypertension in a semiurban community in Nigeria. Eur J Cardiovasc Prev Rehabil. 2008;15:683-687.

12. Oladapo OO, Salako L, Sodiq O, Shoyinka K, Adedapo K, Falase AO. A prevalence of cardiometabolic risk factors among a rural Yoruba South-Western Nigerian population: a population based survey. Cardiovasc J Afr. 2010;21:26-31.

13. Amoah AGB. Hypertension in Ghana: a cross-sectional community prevalence study in Greater Accra. Ethn Dis. 2003;13:310-315.

14. Olatunbosun ST, Kaufman JS, Cooper RS, Bella AF. Hypertension in a Black population: prevalence and biosocial determinants of high blood pressure in a group of urban Nigerians. J Hum Hypertens. 2000;14: 249-257.

15. Kapoor S, Tyagi R, Saluja K, Chaturvedi A, Kapoor AK. Emerging health threats among a primitive tribal group of Central India. Journal of Public Health and Epidemiology. 2010;2:13-19.

16. Ayodele OE, Alebiosu CO, Akinwusi PO, Akinsola A, Mejuini A. Target organ damage and associated clinical conditions in newly diagnosed hypertensives attending a tertiary health facility. Niger J Clin Pract. 2007;10:319-325.

17. Dolea C, Stormont L, Braicheta JM. Evaluated strategies to increase attraction and retention of health workers in remote and rural areas. Bull World Health Organ. 2010;88:379-385.

18. World Health Organization. Physical status: The use and interpretation of anthropometry. Technical report series. Report No 854; Geneva, Switzerland: World Health Organization; 1995. Available from: http:// www.who.int/childgrowth/publications/physical_status/en/index.html. Accessed March 5, 2013.

19. World Health Organization Guidelines subcommittee. 1999 WHO/ ISH guidelines for the management of hypertension. J Hypertens. 1999;17:151-183.

20. Chobanian AV, Bakris GL, Black HR, et al; National High Blood Pressure Education Programme Coordinating Committee: Seventh Report of the Joint National Committee on Prevention, Detection, Evaluation, and Treatment of High Blood Pressure (JNC VII). Hypertension. 2003;42:1206-1252.

21. Addo J, Amoah GB, Koram KA. The changing patterns of hypertension in Ghana: a study of four rural communities in the Ga district. Ethn Dis. 2006;16:894-899.

22. Gupta R. Trends in hypertension epidemiology in India. J Hum Hypertens. 2004;18:73-78.

23. Cooper R, Rotimi C, Ataman S, et al. The prevalence of hypertension in seven populations of West African origin. Am J Public Health. 1997;87: 160-168.

24. Yuvaraj BY, Nagendra Gowda MR, Umakantha AG. Prevalence, awareness, treatment, and control of hypertension in rural areas of Davanagere. Indian J Community Med. 2010;35:138-141.

25. Kokiwar PR, Gupta SS. Prevalence of hypertension in a rural community of Central India. Int J Biol Med Res. 2011;2:950-953.

26. Cappuccio FP, Micah FB, Lynsey E, et al. Prevalence, detection, management, and control of hypertension in Ashanti, West Africa. Hypertension. 2004;43:1017-1022.

27. Wamala JF, Karyabakabo Z, Ndungutse D, Guwatudde D. Prevalence factors associated with Hypertension in Rukungiri District, Uganda a community-based study. Afr Health Sci. 2009;9:153-160.

28. Edwards R, Unwin N, Mugusi F, et al. Hypertension prevalence and care in an urban and rural area of Tanzania. J Hypertens. 2000;18: $145-152$.

29. Addo J, Smeeth L, Leon DA. Hypertension in sub-Saharan Africa: a systematic review. Hypertension. 2007;50:1012-1018.

30. Sayeed MA, Banu A, Khan A, Hussain MZ. Prevalence of diabetes and hypertension in a rural population of Bangladesh. Diabetes Care. 1995;18:555-558. 
31. Will JC. Cigarette smoking and diabetes mellitus: evidence of a positive association from a large prospective cohort study. Int J Epidemiol. 2001;30:540-546.

32. Pobee JO, Larbi EB, Belcher DW, Wurapa FK, Dodu SR. Blood pressure distribution in a rural Ghanaian population. Trans $R$ Soc Trop Med Hyg. 1977;71:66-72.

33. van der Sande MA, Milligan PJ, Nyan OA. Blood pressure patterns and cardiovascular risk factors in rural and urban Gambian communities. J Hum Hypertens. 2000;14:489-496.

34. Mahmood SE, Srivastava A, Shrotriya VP, Shaifali I, Mishra P. National prevalence and epidemiological correlates of hypertension among labour population. J Community Med. 2011;2:43-48.

35. Ghannem H, Fredj AH. Epidemiological transition and cardiovascular risk factors in Tunisia. Rev Epidemiol Sante Publique. 1997;45:286-292. French.

36. Amoah AG. Obesity in adult residents of Accra, Ghana. Ethn Dis. 2003;13(2 Suppl 2):S97-S101.

37. Steyn K, Fourie J, Lombard C, Katzenellenbogen J, Bourne L, Jooste P. Hypertension in the Black community of the Cape Peninsula South Africa. East Afr Med J. 1996;73:758-763.
38. Whelton PK. Epidemiology of hypertension. Lancet. 1994;344: 101-106.

39. Mbanya JCN, Minkoulou EM, Salaha JN, Balkaub B. The prevalence of hypertension in rural and urban Cameroon. Int J Epidemiol. 1998;27: 181-185.

40. Bovet P, Ross AG, Gervasoni JP, Mkamba M, Mtasiwa DM. Distribution of blood pressure, body mass index and smoking habits in the urban population of Dar es Salaam, Tanzania, and associations with socioeconomic status. Int J Epidemiol. 2002;31:240-247.

41. Onal AE, Erbil S, Ozel S, Aciksari K, Tumerdem Y. The prevalence of and risk factors for hypertension in adults living in Istanbul. Blood Press. 2004;13:31-36.

42. Agyemang C. Rural and urban differences in blood pressure and hypertension in Ghana, West Africa. Public Health. 2006;120: 525-533.

43. Amoah AG. Socio-demographic variations in obesity among Ghanaian adults. Public Health Nutr. 2003;6:751-757.
International Journal of General Medicine

\section{Publish your work in this journal}

The International Journal of General Medicine is an international, peer-reviewed open-access journal that focuses on general and internal medicine, pathogenesis, epidemiology, diagnosis, monitoring and treatment protocols. The journal is characterized by the rapid reporting of reviews, original research and clinical studies across all disease areas.

\section{Dovepress}

A key focus is the elucidation of disease processes and management protocols resulting in improved outcomes for the patient.The manuscript management system is completely online and includes a very quick and fair peer-review system. Visit http://www.dovepress.com/ testimonials.php to read real quotes from published authors. 